\title{
GLOBAL ASYMPTOTICS TOWARD THE RAREFACTION WAVE FOR SOLUTIONS OF VISCOUS $p$-SYSTEM WITH BOUNDARY EFFECT
}

\author{
BY \\ AKITAKA MATSUMURA (Department of Mathematics, Graduate School of Science, Osaka \\ University, Japan) \\ AND \\ KENJI NISHIHARA (School of Political Science and Economics, Waseda University, Tokyo, \\ Japan)
}

Dedicated to Professor Kiyoshi Mochizuki on his sixtieth birthday

\begin{abstract}
The initial-boundary value problem on the half-line $\mathbf{R}_{+}{ }^{*}=(0, \infty)$ for a system of barotropic viscous flow $v_{t}-u_{x}=0, u_{t}+p(v)_{x}=\mu\left(\frac{u_{x}}{v}\right)_{x}$ is investigated, where the pressure $p(v)=v^{-\gamma}(\gamma \geq 1)$ for the specific volume $v>0$. Note that the boundary value at $x=0$ is given only for the velocity $u$, say $u_{-}$, and that the initial data $\left(v_{0}, u_{0}\right)(x)$ have the constant states $\left(v_{+}, u_{+}\right)$at $x=+\infty$ with $v_{0}(x)>0$, $v_{+}>0$. If $u_{-}<u_{+}$, then there is a unique $v_{-}$such that $\left(v_{+}, u_{+}\right) \in R_{2}\left(v_{-}, u_{-}\right)$ (the 2-rarefaction curve) and hence there exists the 2-rarefaction wave $\left(v_{2}^{R}, u_{2}^{R}\right)(x / t)$ connecting $\left(v_{-}, u_{-}\right)$with $\left(v_{+}, u_{+}\right)$. Our assertion is that, if $u_{-}<u_{+}$, then there exists a global solution $(v, u)(t, x)$ in $C^{0}\left([0, \infty) ; H^{1}\left(\mathbf{R}_{+}\right)\right)$, which tends to the 2-rarefaction wave $\left.\left(v_{2}^{R}, u_{2}^{R}\right)(x / t)\right|_{x \geq 0}$ as $t \rightarrow \infty$ in the maximum norm, with no smallness condition on $\left|u_{+}-u_{-}\right|$and $\left\|\left(v_{0}-v_{+}, u_{0}-u_{+}\right)\right\|_{H^{1}}$, nor restriction on $\gamma(\geq 1)$. A similar result to the corresponding Cauchy problem is also obtained. The proofs are given by an elementary $L^{2}$-energy method.
\end{abstract}

Received February 15, 1998.

2000 Mathematics Subject Classification. Primary 35B40, 35L65, 76N10.

Research by the second author was supported in part by Waseda University Grant for Special Research Project 97A-242. 
1. Introduction. We consider the initial-boundary value problem on $\mathbf{R}_{+}=(0, \infty)$ for a system of the barotropic viscous flow in the Lagrangean coordinate:

$$
\left\{\begin{array}{l}
v_{t}-u_{x}=0, \quad(t, x) \in \mathbf{R}_{+} \times \mathbf{R}_{+} \\
u_{t}+p(v)_{x}=\mu\left(\frac{u_{x}}{v}\right)_{x} \\
u(t, 0)=u_{-} \\
(v, u)(0, x)=\left(v_{0}, u_{0}\right)(x), \quad v_{0}(x)>0
\end{array}\right.
$$

where $v(>0)$ is the specific volume, $u$ is the velocity, $\mu(>0)$ is the constant coefficient of viscosity and $p$ is the pressure given by

$$
p(v)=v^{-\gamma}, \quad \gamma \geq 1
$$

The initial data is assumed to tend to the constant state as $x \rightarrow+\infty$ :

$$
\lim _{x \rightarrow \infty}\left(v_{0}, u_{0}\right)(x)=\left(v_{+}, u_{+}\right), \quad v_{+}>0 .
$$

The compatibility condition

$$
u_{0}(0)=u_{-}
$$

is also assumed. Note that the boundary condition is posed only on $u$.

Our main concern is to investigate the large-time behavior of the solution $(v, u)(t, x)$ of (1.1) when

$$
u_{-}<u_{+} \text {. }
$$

When $u_{+}<u_{-}=0$, see Matsumura and Mei [6]. Here we note that the condition $u_{-}=0$ is not necessarily assumed. However, from the physical background of our problem we have $u_{-}=0$ in mind, which means the one-dimensional viscous flow with fixed boundary at $x=0$ in the Eulerian coordinate. For the problem of a single equation in the quarter plane in $(x, t)$, see [3], [4], [5] and the references therein.

To state our result, we now mention the corresponding Riemann problem on $\mathbf{R}=$ $(-\infty, \infty)$ for given constant states $\left(v_{ \pm}, u_{ \pm}\right), v_{ \pm}>0$ :

$$
\left\{\begin{array}{l}
v_{t}-u_{x}=0, \quad(t, x) \in \mathbf{R}_{+} \times \mathbf{R} \\
u_{t}+p(v)_{x}=0 \\
(v, u)(0, x)=\left(v_{0}^{R}, u_{0}^{R}\right)(x)= \begin{cases}\left(v_{-}, u_{-}\right) & x<0 \\
\left(v_{+}, u_{+}\right) & x>0\end{cases}
\end{array}\right.
$$

As is well known, if $\left(v_{+}, u_{+}\right) \in R_{i}\left(v_{-}, u_{-}\right)$(resp. $\left.S_{i}\left(v_{-}, u_{-}\right)\right)$for $i=1,2$, then (1.6) admits a weak entropy solution $\left(v_{i}^{R}, u_{i}^{R}\right)(x / t)$ called the $i$-rarefaction wave (resp. $\left(v_{i}^{S}, u_{i}^{S}\right)\left(x-s_{i} t\right)$ is called the $i$-shock wave), where for a suitable neighborhood $\omega$ of $\left(v_{-}, u_{-}\right)$in $\mathbf{R}_{(v, u)}^{2}$

$$
R_{i}\left(v_{-}, u_{-}\right)=\left\{(v, u) \in \omega \mid u=u_{-}-\int_{v_{-}}^{v} \lambda_{i}(s) d s, \quad u \geq u_{-}\right\}
$$


with the eigenvalues $\lambda_{i}(v)=(-1)^{i} \sqrt{-p^{\prime}(v)}$ of $\left(\begin{array}{cc}0 & -1 \\ p^{\prime}(v) & 0\end{array}\right)$, and

$$
\begin{array}{r}
S_{i}\left(v_{-}, u_{-}\right)=\left\{(v, u) \in \omega \mid u-u_{-}=-\sqrt{\left|\left(v-v_{-}\right)\left(p(v)-p\left(v_{-}\right)\right)\right|}\right. \\
\left.(-1)^{i}\left(v-v_{-}\right) \geq 0\right\}
\end{array}
$$

with the Rankine-Hugoniot conditions $-s_{i}\left(v_{+}-v_{-}\right)-\left(u_{+}-u_{-}\right)=0,-s_{i}\left(u_{+}-u_{-}\right)+$ $p\left(v_{+}\right)-p\left(v_{-}\right)=0$.

Since there is a boundary at $x=0$ in our problem, the backward flow reflects at the boundary and the total flow is eventually expected to move forward and behave as the 2 -rarefaction wave for large time because $u_{+}>u_{-}$. Thus, we reach the conjecture that the solution $(v, u)$ of $(1.1)$ behaves as

$$
\left(V_{2}^{R}, U_{2}^{R}\right)(x / t):=\left.\left(v_{2}^{R}, u_{2}^{R}\right)(x / t)\right|_{x \geq 0},
$$

where

$$
\left\{\begin{array}{l}
\left(v_{2}^{R}, u_{2}^{R}\right)(x / t) \text { is the 2-rarefaction wave connecting two } \\
\text { constant states }\left(v_{-}, u_{-}\right) \text {to }\left(v_{+}, u_{+}\right) \text {, with } v_{-}(>0) \\
\text { uniquely determined by }\left(v_{+}, u_{+}\right) \in R_{2}\left(v_{-}, u_{-}\right) \text {for given } \\
\text { constants } v_{+}(>0) \text { and } u_{ \pm} .
\end{array}\right.
$$

For simplicity we call (1.8) the "rarefaction wave". Recently, the stability of the weak rarefaction wave $\left(V_{2}^{R}, U_{2}^{R}\right)(x / t)$ has been shown by Pan, Liu, and Nishihara [9].

Our purpose is to show the large-time behavior of the solution $(v, u)$ of $(1.1)$ without restrictions on $\left|u_{+}-u_{-}\right|,\left\|v_{0}-v_{+}, u_{0}-u_{+}\right\|_{H^{1}\left(\mathbf{R}_{+}\right)}$, and $\gamma(\geq 1)$.

THEOREM 1.1. For given constants $v_{+}, u_{ \pm}$satisfying (1.5), suppose that $\left(v_{0}-v_{+}, u_{0}-\right.$ $\left.u_{+}\right) \in H^{1}\left(\mathbf{R}_{+}\right)$with (1.4). Then there exists a unique global solution $(v, u)(t, x)$ of $(1.1)$ in $C^{0}\left([0, \infty) ; H^{1}\left(\mathbf{R}_{+}\right)\right)$that satisfies

$$
\sup _{\mathbf{R}_{+}}\left|\left(v-V_{2}^{R}, u-U_{2}^{R}\right)(t, x)\right| \rightarrow 0 \quad \text { as } t \rightarrow \infty,
$$

where $\left(V_{2}^{R}, U_{2}^{R}\right)$ is given by (1.8) and (1.9).

We now mention the corresponding Cauchy problem for given constant states $\left(v_{ \pm}, u_{ \pm}\right)$, $v_{ \pm}>0$

$$
\left\{\begin{array}{l}
v_{t}-u_{x}=0, \quad(t, x) \in \mathbf{R}_{+} \times \mathbf{R}, \\
u_{t}+p(v)_{x}=\mu\left(\frac{u_{x}}{v}\right)_{x}, \\
(v, u)(0, x)=\left(v_{0}, u_{0}\right)(x) \rightarrow\left(v_{ \pm}, u_{ \pm}\right), \quad x \rightarrow \pm \infty .
\end{array}\right.
$$

Here we assume that

$$
\begin{array}{r}
\left(v_{+}, u_{+}\right) \in R_{R}\left(v_{-}, u_{-}\right)=\left\{(v, u) \in \omega \mid u \geq u_{-}-\int_{v_{-}}^{v} \lambda_{1}(s) d s,\right. \\
\left.u \geq u_{-}-\int_{v_{-}}^{v} \lambda_{2}(s) d s, \quad u \geq u_{-}\right\}
\end{array}
$$


and there exists a unique state $(\bar{v}, \bar{u}) \in R_{1}\left(v_{-}, u_{-}\right)$such that $\left(v_{+}, u_{+}\right) \in R_{2}(\bar{v}, \bar{u})$, and that the Riemann problem (1.6) admits a rarefaction wave connecting $\left(v_{-}, u_{-}\right)$with $\left(v_{+}, u_{+}\right)$:

$$
\left(v^{R}, u^{R}\right)(x / t):=\left(v_{1}^{R}+v_{2}^{R}-\bar{v}, u_{1}^{R}+u_{2}^{R}-\bar{u}\right)(x / t)
$$

where $\left(v_{1}^{R}, u_{1}^{R}\right)$ is the 1-rarefaction wave connecting $\left(v_{-}, u_{-}\right)$with $(\bar{v}, \bar{u})$, and $\left(v_{2}^{R}, u_{2}^{R}\right)$ is the 2-rarefaction wave connecting $(\bar{v}, \bar{u})$ with $\left(v_{+}, u_{+}\right)$.

In [8] the authors showed that a unique solution $(v, u)$ of $(1.11)$ behaves as $\left(v^{R}, u^{R}\right)(x / t)$ in (1.13) provided that

$$
1 \leq \gamma \leq 2
$$

Our result here is that the same theorem as in [8] holds even for any $\gamma \geq 1$.

ThEOREM 1.2. For given constant states $\left(v_{ \pm}, u_{ \pm}\right), v_{ \pm}>0$, with $\left(v_{+}, u_{+}\right) \in R_{R}\left(v_{-}, u_{-}\right)$, suppose that the Riemann problem (1.6) admits a unique continuous solution $\left(v^{R}, u^{R}\right)(x / t)$ in (1.13). If $\left(v_{0}-v_{0}^{R}, u_{0}-u_{0}^{R}\right) \in L^{2}(\mathbf{R}),\left(v_{0 x}, u_{0 x}\right) \in L^{2}(\mathbf{R})$ with $v_{0}(x)>0$, then the Cauchy problem (1.11) has a unique global solution $(v, u)$ in time satisfying

$$
\begin{gathered}
\left(v-v^{R}, u-u^{R}\right) \in C^{0}\left([0, \infty) ; L^{2}\right) \cap L^{\infty}\left(0, \infty ; L^{2}\right), \\
(v, u)_{x} \in C^{0}\left([0, \infty) ; L^{2}\right) \cap L^{\infty}\left(0, \infty ; L^{2}\right) \cap L^{2}((0, \infty) \times \mathbf{R}), \\
u_{x x} \in L^{2}((0, \infty) \times \mathbf{R}),
\end{gathered}
$$

and

$$
\lim _{t \rightarrow \infty} \sup _{\mathbf{R}}\left|\left(v-v^{R}, u-u^{R}\right)(t, x)\right|=0 .
$$

The proof of Theorem 1.2 is almost the same as in the previous work [7], but more delicate estimates are necessary to remove (1.14), which will be given in the same way as the estimates in the proof of Theorem 1.1. Especially, see Step 3 in Sec. 4. Therefore, in the sections below we devote ourselves to the initial-boundary value problem and so the proof of Theorem 1.1. Our plan of this paper is as follows. In the next two sections we construct a smooth rarefaction wave $(V, U)(t, x)$ of $\left(V_{2}^{R}, U_{2}^{R}\right)(x / t)=\left.\left(v_{2}^{R}, u_{2}^{R}\right)(x / t)\right|_{x \geq 0}$, and reformulate our problem to that of the perturbation $(\phi, \psi)$ from $(V, U)(t, x)$, for which the $L^{2}$-energy method will be employed. In the last section we establish the a priori estimates.

2. Smooth rarefaction wave. Similar to [7], [8] we start with the Riemann problem on $\mathbf{R}=(-\infty, \infty)$ for the typical Burgers equation:

$$
\left\{\begin{array}{l}
w_{t}^{R}+w^{R} w_{x}^{R}=0, \quad(t, x) \in \mathbf{R}_{+} \times \mathbf{R}, \\
w^{R}(0, x)=w_{0}^{R}(x)= \begin{cases}w_{-} & x<0 \\
w_{+} & x>0\end{cases}
\end{array}\right.
$$


with $w_{-}<w_{+}$. The weak solution of (2.1) with the entropy condition is a rarefaction wave $w^{R}(x / t)$ connecting $w_{-}$with $w_{+}$:

$$
w^{R}(x / t)= \begin{cases}w_{-} & x \leq w_{-} t \\ x / t & w_{-} t<x<w_{+} t \\ w_{+} & w_{+} t \leq x\end{cases}
$$

In our problem the smooth rarefaction wave that is approximate to $w^{R}(x / t)$ is given by a unique solution of

$$
\left\{\begin{array}{l}
w_{t}+w w_{x}=0 \\
w(0, x)=w_{0}(x):=\hat{w}+\tilde{w} \cdot \kappa_{q} \int_{0}^{\varepsilon x}\left(1+y^{2}\right)^{-q} d y
\end{array}\right.
$$

where $\hat{w}=\left(w_{+}+w_{-}\right) / 2, \tilde{w}=\left(w_{+}-w_{-}\right) / 2, \varepsilon>0$ is a small constant to be determined later, and $\kappa_{q}$ is a constant satisfying $\kappa_{q} \int_{0}^{\infty}\left(1+y^{2}\right)^{-q} d y=1$ for a large constant $q(>3 / 2)$ also to be determined later.

We state the properties of $w$ of $(2.3)$ when $w_{+}>w_{-}>0$, since the forward rarefaction wave will be considered in our initial-boundary value problem.

LEMma 2.1. Let $w_{+}>w_{-}>0$. Then, the unique, smooth and time-global solution $w(t, x)$ satisfies the following properties:

(i) $w_{-}<w(t, x)<w_{+}, w_{x}(t, x)>0$.

(ii) For any $p(1 \leq p \leq \infty)$ there exists a constant $C_{p, q}$ such that

$$
\begin{aligned}
\left\|w_{x}(t, \cdot)\right\|_{L^{P}} & \leq C_{p, q} \min \left(\varepsilon^{1-1 / p} \tilde{w}, \tilde{w}^{1 / p} t^{-1+1 / p}\right), \\
\left\|w_{x x}(t, \cdot)\right\|_{L^{p}} & \leq \min \left(\varepsilon^{2-1 / p} \tilde{w}, \varepsilon^{(1-1 /(2 q))(1-1 / p)} \tilde{w}^{-(p-1) /(2 p q)} t^{-1-(p-1) /(2 p q)}\right) .
\end{aligned}
$$

(iii) For a positive constant $C_{q}$ and any $x \leq 0$,

$$
\begin{aligned}
& 0<w(t, x)-w_{-} \leq C_{q}\left(1+(\varepsilon x)^{2}\right)^{-q / 3}\left(1+\left(\varepsilon w_{-} t\right)^{2}\right)^{-q / 3} \\
& 0<w_{x}(t, x) \leq C_{q} \varepsilon \tilde{w}\left(1+(\varepsilon x)^{2}\right)^{-q / 2}\left(1+\left(\varepsilon w_{-} t\right)^{2}\right)^{-q / 2} .
\end{aligned}
$$

(iv) $\lim _{t \rightarrow \infty} \sup _{\mathbf{R}}\left|w(t, x)-w^{R}(x / t)\right|=0$.

For the proof see Matsumura and Nishihara [7, 8].

For the positive eigenvalue $\lambda_{2}(v)=\sqrt{-p^{\prime}(v)}$ and the constant states $\left(v_{ \pm}, u_{ \pm}\right)$with $\left(v_{+}, u_{+}\right) \in R_{2}\left(v_{-}, u_{-}\right)$, define $(\bar{V}, \bar{U})(t, x)$ by

$$
\lambda_{2}(\bar{V})=w(t, x), \quad \bar{U}=u_{-}-\int_{v_{-}}^{\bar{V}(t, x)} \lambda_{2}(s) d s
$$

together with $\lambda_{2}\left(v_{ \pm}\right)=w_{ \pm}$, along the standard way of the construction of a 2-rarefaction wave:

$$
\lambda_{2}\left(v^{R}(\xi)\right)=w^{R}(\xi), \quad u^{R}(\xi)=u_{-}-\int_{v_{-}}^{v^{R}(\xi)} \lambda_{2}(s) d s, \quad \xi=x / t .
$$

Then, $(\bar{V}, \bar{U})(t, x)$ satisfies

$$
\left\{\begin{array}{l}
\bar{V}_{t}-\bar{U}_{x}=0 \\
\bar{U}_{t}+p(\bar{V})_{x}=0
\end{array}\right.
$$


Moreover, the properties of $w$ shift to those of $(\bar{V}, \bar{U})$.

Lemma 2.2. Let $\delta=\left|v_{+}-v_{-}\right|+\left|u_{+}-u_{-}\right|$. Then $(\bar{V}, \bar{U})$ satisfies the following:

(i) $\bar{V}_{t}=\bar{U}_{x}>0$.

(ii) For some positive constant $C$,

$$
\left|\bar{V}_{x}\right| \leq C \bar{V}_{t}, \quad \bar{V}_{t} \leq C \varepsilon \delta .
$$

(iii) For any $p(1 \leq p \leq \infty)$ there exists a constant $C_{p, q}$ such that

$$
\begin{gathered}
\left\|\left(\bar{V}_{x}, \bar{U}_{x}\right)(t, \cdot)\right\|_{L^{p}} \leq C_{p, q} \min \left(\delta \varepsilon^{1-1 / p}, \delta^{1 / p}(1+t)^{-1+1 / p}\right) \\
\left\|\left(\bar{V}_{x x}, \bar{U}_{x x}\right)(t, \cdot)\right\|_{L^{p}} \leq \min \left\{\delta^{-(p-1) /(2 p q)} \varepsilon^{(1-1 /(2 q))(1-1 / p)}(1+t)^{-1-(p-1) /(2 p q)}\right. \\
\left.+\delta^{1 / p}(1+t)^{-2+1 / p}\right\} .
\end{gathered}
$$

Especially, when $p>1$

$$
\int_{0}^{\infty}\left\|\left(\bar{V}_{x x}, \bar{U}_{x x}\right)(t, \cdot)\right\|_{L^{p}} d t \leq C_{p, q} \delta^{-(p-1) /(2 p q)} .
$$

(iv) For a positive constant $C_{q}$ and any $x \leq 0$

$$
\begin{aligned}
& 0<\left(v_{-}-\bar{V}, \bar{U}-u_{-}\right)(t, x) \leq C_{q} \delta\left(1+(\varepsilon t)^{2}\right)^{-q / 3}, \\
& 0<\left(-\bar{V}_{x}, \bar{U}_{x}\right)(t, x) \leq C_{q} \varepsilon \delta\left(1+(\varepsilon t)^{2}\right)^{-q / 2} .
\end{aligned}
$$

(v) $\lim _{t \rightarrow \infty} \sup _{\mathbf{R}}\left|(\bar{V}, \bar{U})(t, x)-\left(v^{R}, u^{R}\right)(x / t)\right|=0$.

We now set

$$
(V, U)(t, x):=(V, U)\left(t, x ; \varepsilon, q, t_{0}\right)=\left.(\bar{V}, \bar{U})\left(t+t_{0}, x\right)\right|_{x \geq 0}
$$

as a smooth approximation to $\left(V_{2}^{R}, U_{2}^{R}\right)$ in (1.8), where the small parameter $\varepsilon>0$ and large ones $q(>3 / 2), t_{0}>0$ will be determined later.

The property (2.6) is important to obtain the global result. In [9] the choice of $w_{0}(x)$ in (2.3) eliminates the boundary-layer, but no property such as (2.6) detains the result weaker. Instead, we have the boundary-layer such as (iv) in Lemma 2.2, for which much more delicate estimates of not only the nonlinear terms but also the values from the boundary are necessary.

3. Reformulation of the problem. We rewrite (1.1) by a system of the perturbation $(\phi, \psi)$ from the smooth rarefaction wave $(V, U)$ in $(2.7)$ :

$$
\left\{\begin{array}{l}
\phi_{t}-\psi_{x}=0, \quad(t, x) \in \mathbf{R}_{+} \times \mathbf{R}_{+} \\
\psi_{t}+(p(V+\phi)-p(V))_{x}-\mu\left(\frac{u_{x}}{v}-\frac{U_{x}}{V}\right)_{x}=G_{x}, \\
\psi(t, 0)=b(t):=u_{-}-U(t, 0), \\
(\phi, \psi)(0, x)=\left(\phi_{0}, \psi_{0}\right)(x):=\left(v_{0}(x)-V(0, x), u_{0}(x)-U(0, x)\right),
\end{array}\right.
$$

where

$$
G_{x}=\mu\left(\frac{U_{x}}{V}\right)_{x}
$$


Though (3.1) is a system of $(\phi, \psi)$, we often use the notation $v=V+\phi, u=U+\psi$. By virtue of Lemma 2.2 and the definition (2.7),

$$
\begin{gathered}
0>b(t) \geq-C\left(1+\left(\varepsilon t_{0}\right)^{2}\right)^{-q / 6}\left(1+(\varepsilon t)^{2}\right)^{-q / 6}, \\
0<b^{\prime}(t)=-U_{t}(t, 0) \leq C \varepsilon\left(1+\left(\varepsilon t_{0}\right)^{2}\right)^{-q / 4}\left(1+(\varepsilon t)^{2}\right)^{-q / 4},
\end{gathered}
$$

and

$$
\int_{0}^{\infty}\left\|G_{x}(t, \cdot)\right\| d t \leq C .
$$

Here and after, we denote several generic constants depending on $a, b, \ldots$ by $C_{a, b, \ldots}$ or simply by $C$ without confusion, and also by $\|\cdot\|$ denote the norm in the Lebesque space $L^{2}=L^{2}\left(\mathbf{R}_{+}\right)$.

We look for the solution $(\phi, \psi)$ of $(3.1)$ in the solution space $X(0, \infty):=\bigcup_{0<m<M<\infty}$ $\bigcap_{0 \leq t_{1}<t_{2}<\infty} X_{m, M}\left(t_{1}, t_{2}\right)$, where for $t_{1}, t_{2}\left(0 \leq t_{1}<t_{2}<\infty\right)$ and $m, M(0<m<M<$ $\infty)$

$$
\begin{aligned}
X_{m, M}\left(t_{1}, t_{2}\right)= & \left\{(\phi, \psi) \mid(\phi, \psi) \in C^{0}\left(\left[t_{1}, t_{2}\right] ; H^{1}\right),\right. \\
& \phi_{x} \in L^{2}\left(t_{1}, t_{2} ; L^{2}\right), \quad \psi_{x} \in L^{2}\left(t_{1}, t_{2} ; H^{1}\right), \\
& \psi\left(t_{1}, 0\right)=b\left(t_{1}\right), \sup _{t_{1} \leq t \leq t_{2}}\|(\phi, \psi)(t)\|_{1} \leq M, \\
& \text { and } \left.\inf _{t_{1} \leq t \leq t_{2}} \inf _{\mathbf{R}}(V(t, x)+\phi(t, x)) \geq m\right\} .
\end{aligned}
$$

Here, $H^{k}(k \geq 0)$ denotes the usual Sobolev space on $\mathbf{R}_{+}$of order $k$ with the norm $\|\cdot\|_{k}$. In particular, $H^{0}=L^{2}$ and $\|\cdot\|_{0}=\|\cdot\|$.

For the proof of Theorem 1.1 it suffices to show

Theorem 3.1. Suppose that $\left(\phi_{0}, \psi_{0}\right) \in H^{1}$. Then, there exist a unique solution $(\phi, \psi) \in$ $X(0, \infty)$ and a positive constant $C_{0}$ for a suitably small $\varepsilon>0$ and suitably large $q, t_{0}>0$ that satisfy

$$
\begin{gathered}
C_{0}^{-1} \leq V+\phi \leq C_{0}, \\
\sup _{t \geq 0}\|(\phi, \psi)(t)\|_{1}^{2}+\int_{0}^{\infty}\left(\left\|\sqrt{V_{t}} \phi(\tau)\right\|^{2}+\left\|\phi_{x}(\tau)\right\|^{2}+\left\|\psi_{x}(\tau)\right\|_{1}^{2}\right) d \tau \\
\leq C_{0}\left(\left\|\phi_{0}, \psi_{0}\right\|_{1}^{2}+1\right) .
\end{gathered}
$$

Theorem 3.1 is obtained by the combination of the local existence with the a priori estimates. To state the local existence theorem we consider the initial-boundary value problem with initial time $\tau(\geq 0)$ :

$$
\left\{\begin{array}{l}
\phi_{t}-\psi_{x}=0, \quad t>\tau, x \in \mathbf{R} \\
\psi_{t}-\mu\left(\frac{\psi_{x}}{V+\phi}\right)_{x}=F\left(\phi, \phi_{x}\right)_{x} \\
\psi(t, 0)=b(t), \quad t \geq \tau \\
(\phi, \psi)(\tau, x)=\left(\phi_{\tau}, \psi_{\tau}\right)(x), \quad \psi_{\tau}=b(\tau)
\end{array}\right.
$$


instead of (3.1), where

$$
F_{x}=-(p(V+\phi)-p(V))_{x}+\mu\left(\frac{U_{x}}{V+\phi}-\frac{U_{x}}{V}\right)_{x}+G_{x}
$$

Then, the local existence theorem is given as follows:

Proposition 3.1. For any given $\left(\phi_{\tau}, \psi_{\tau}\right) \in H^{1}$ with $\psi_{\tau}(0)=b(\tau),\left\|\phi_{\tau}, \psi_{\tau}\right\|_{1} \leq M$ and $\inf _{\mathbf{R}_{+}}\left(V(\tau, x)+\psi_{\tau}(x)\right) \geq m$, there exists a constant $t_{0}=t_{0}(m, M)$ independent of $\tau$ such that there is a unique solution $(\phi, \psi)$ of $(3.7)$ in $X_{\frac{m}{2} .2 M}\left(\tau, \tau+t_{0}\right)$.

The linear initial-boundary value problem on the half-line for the parabolic equation

$$
\left\{\begin{array}{l}
\psi_{t}-\left(a(t, x) \psi_{x}\right)_{x}=f(t, x), \quad t>\tau, x \in \mathbf{R}_{+} \\
\psi(t, 0)=b(t), \quad t \geq \tau \\
\psi(\tau, x)=\psi_{\tau}(x), \quad x \in \mathbf{R}_{+}
\end{array}\right.
$$

has a smooth solution $\psi(t, x)$. For example, see [2, Chapt. 4]. Therefore, the approximate solution $\left\{\left(\phi^{(n)}, \psi^{(n)}\right)\right\}=\left\{\left(\phi_{(k)}^{(n)}, \psi_{(k)}^{(n)}\right)\right\}$ is taken by

$$
\left(\phi^{(0)}, \psi^{(0)}\right)(t, x)=\left(\phi_{\tau(k)}, \psi_{\tau(k)}\right)(x) \in H^{l} \quad(l \gg 1)
$$

and

$$
\left\{\begin{array}{l}
\psi_{t}^{(n+1)}-\mu\left(\frac{\psi_{x}^{(n+1)}}{V+\phi^{(n)}}\right)_{x}=F\left(\phi^{(n)}, \phi_{x}^{(n)}\right), \\
\text { with }\left.\psi^{(n+1)}\right|_{t=\tau}=\psi_{\tau(k)}(x),\left.\quad \psi^{(n+1)}\right|_{x=0}=b(t), \\
\phi^{(n+1)}(t, x)=\phi_{\tau(k)}+\int_{\tau}^{t} \psi_{x}^{(n+1)}(\tau, x) d \tau,
\end{array}\right.
$$

where as $k \rightarrow \infty$

$$
\left(\phi_{\tau(k)}, \psi_{\tau(k)}\right) \rightarrow\left(\phi_{\tau}, \psi_{\tau}\right) \quad \text { in } H^{1}
$$

with $\left\|\phi_{\tau(k)}, \psi_{\tau(k)}\right\|_{1} \leq \frac{3}{2} M$ and $\inf _{\mathbf{R}_{+}}\left(V(\tau, x)+\phi_{\tau(k)}\right) \geq \frac{3}{4} m$ for any $k \geq 1$. Then we have the Cauchy sequence $\left(\phi_{(k)}^{(n)}, \psi_{(k)}^{(n)}\right)$ in $n$ and $\left(\phi_{(k)}, \psi_{(k)}\right):=\lim _{n \rightarrow \infty}\left(\phi_{(k)}^{(n)}, \psi_{(k)}^{(n)}\right)$ in $X_{\frac{m}{2}, 2 M}\left(\tau, \tau+t_{0}\right)$. Finally, taking $k \rightarrow \infty$, we obtain Proposition 3.1 , though the details are omitted.

Corresponding to Proposition 3.1, the a priori estimates are mentioned as follows.

Proposition 3.2 (A Priori Estimates). Let $(\phi, \psi)(t, x)$ be a solution of $(3.1)$ in $X(0, T)$ for some $T>0$. Then there is a constant $C_{1}$ independent of $T$ such that for $0 \leq t \leq T$,

$$
\begin{gathered}
C_{1}^{-1} \leq V(t, x)+\phi(t, x) \leq C_{1}, \\
\|(\phi, \psi)(t)\|_{1}^{2}+\int_{0}^{t}\left(\left\|\sqrt{V_{t}} \phi(\tau)\right\|^{2}+\left\|\phi_{x}(\tau)\right\|^{2}+\left\|\psi_{x}(\tau)\right\|_{1}^{2}\right) d \tau \\
\leq C\left(\left\|\phi_{0}, \psi_{0}\right\|_{1}^{2}+1\right) .
\end{gathered}
$$


4. A priori estimates. It suffices to establish the a priori estimates for a sufficiently smooth solution $(\phi, \psi)(t, x) \in X(0, T)$, because the arguments on the molifier can be applied. Note that it is important to obtain (3.10). In a series of several steps we show (3.10), which will be done in Step 5, and so (3.11).

Step 1. Multiplying $(3.1)_{2}$ (second equation of $\left.(3.1)\right)$ by $\psi$ and using $(3.1)_{1}$, we have a divergence form

$$
\begin{gathered}
\left\{\frac{1}{2} \psi^{2}+\Phi(v, V)\right\}_{t}+\left\{(p(V+\phi)-p(V)) \psi-\mu\left(\frac{U_{x}+\psi_{x}}{v}-\frac{U_{x}}{V}\right) \psi\right\}_{x} \\
+\mu \frac{\psi_{x}^{2}}{v}-\mu \frac{V_{t} \psi_{x} \phi}{v V}+\left(p(V+\phi)-p(V)-p^{\prime}(V) \phi\right) V_{t}=G_{x} \cdot \psi,
\end{gathered}
$$

where

$$
\Phi(v, V)=p(V) \phi-\int_{V}^{V+\phi} p(s) d s
$$

If we put $p(V+\phi)-p(V)-p^{\prime}(V) \phi=f(v, V) \phi^{2}$ and regard the last three terms in the left-hand side of (4.1) as a quadratic equation of $\sqrt{\mu} \frac{\psi_{x}}{\sqrt{v}}$ and $\sqrt{f(v, V) V_{t}} \phi$, then the discriminant is

$$
D=\mu \frac{V_{t}}{V^{2} v f(v, V)}-4
$$

Since $f(v, V)=O\left(v^{-\gamma}\right)$ as $v \rightarrow 0+$ and $f(v, V)=O\left(v^{-1}\right)$ as $v \rightarrow \infty, 0 \leq \frac{\mu}{V^{2} v f(v, V)} \leq$ $C<+\infty$ for any $v(0<v<\infty)$. Noting that $0<V_{t}<C \varepsilon$ by Lemma 2.2, we fix $\varepsilon>0$ so small that $D<0$. (Later, it is necessary to take $\varepsilon$ smaller as needed. See Step 3.) Thus, integrating (4.1) over $[0, t] \times \mathbf{R}_{+}$, we have the first lemma.

LEMMA 4.1. It follows that

$$
\begin{aligned}
& \frac{1}{2}\|\psi(t)\|^{2}+\int_{0}^{\infty} \Phi(v, V)(t, x) d x \\
& \quad+C^{-1} \int_{0}^{t} \int_{0}^{\infty}\left\{\left(p(V+\phi)-p(V)-p^{\prime}(V) \phi\right) V_{t}+\left|\frac{\phi \psi_{x} V_{t}}{v V}\right|+\frac{\psi_{x}^{2}}{v}\right\} d x d \tau \\
& \leq C\left\|\phi_{0}, \psi_{0}\right\|^{2}+\int_{0}^{t}\left\|G_{x}(\tau)\right\|\|\psi(\tau)\| d \tau \\
& \quad+\left.\int_{0}^{t}\left\{(p(V+\phi)-p(V)) \psi-\mu\left(\frac{U_{x}+\psi_{x}}{v}-\frac{U_{x}}{V}\right) \psi\right\}\right|_{x=0} d \tau
\end{aligned}
$$


If we adopt a new variable $\tilde{v}=v / V$ and use $V_{t}=U_{x}, \phi_{t}=\psi_{x}$, then (4.3) is rewritten as

$$
\begin{aligned}
\frac{1}{2} \| \psi(t) & \|^{2}+\int_{0}^{\infty} V(t, x)^{1-\gamma} \widetilde{\Phi}(\tilde{v}(t, x)) d x \\
& +C^{-1} \int_{0}^{t} \int_{0}^{\infty}\left\{\frac{V_{t}}{V^{\gamma}}\left(\frac{1}{\tilde{v}^{\gamma}}-1+\gamma(\tilde{v}-1)\right)+\left|\frac{\phi \psi_{x} V_{t}}{v V}\right|+\frac{\psi_{x}^{2}}{v}\right\} d x d \tau \\
\leq & C\left\|\phi_{0}, \psi_{0}\right\|^{2}+\int_{0}^{t}\left\|G_{x}(\tau)\right\|\|\psi(\tau)\| d \tau \\
& +\left.\int_{0}^{t}\left\{(p(V+\phi)-p(V)) \psi-\mu\left(\frac{U_{x}+\psi_{x}}{v}-\frac{U_{x}}{V}\right) \psi\right\}\right|_{x=0} d \tau,
\end{aligned}
$$

where

$$
\widetilde{\Phi}(\eta)= \begin{cases}\eta-1-\log \eta, & \gamma=1 \\ \eta-1-\left(\eta^{1-\gamma}-1\right) /(\gamma-1), & \gamma>1\end{cases}
$$

Step 2. We rewrite (3.1) to the form of $\tilde{v}$ :

$$
\left(\mu \frac{\tilde{v}_{x}}{\tilde{v}}-\psi\right)_{t}+\frac{\gamma \tilde{v}_{x}}{V^{\gamma} \tilde{v}^{\gamma+1}}+\frac{\gamma V_{x}}{V^{\gamma+1}}\left(\frac{1}{\tilde{v}^{\gamma}}-1\right)=-G_{x}
$$

Multiply (4.5) by $\tilde{v}_{x} / \tilde{v}$ to get

$$
\begin{aligned}
& \left\{\frac{\mu}{2}\left(\frac{\tilde{v}_{x}}{\tilde{v}}\right)^{2}-\psi \frac{\tilde{v}_{x}}{\tilde{v}}\right\}_{t}+\left\{\left(\frac{\psi_{t}}{v}+\left(\frac{1}{v}-\frac{1}{V}\right) V_{t}\right) \psi\right\}_{x} \\
& \quad+\frac{\gamma \tilde{v}_{x}^{2}}{V^{\gamma} \tilde{v}^{\gamma+2}}+\frac{\gamma V_{x}}{V^{\gamma+1}}\left(\frac{1}{\tilde{v}^{\gamma}}-1\right) \frac{\tilde{v}_{x}}{\tilde{v}}=-G_{x} \cdot \frac{\tilde{v}_{x}}{\tilde{v}}+\frac{\psi_{x}^{2}}{v}-\frac{\phi \psi_{x} V_{t}}{v V} .
\end{aligned}
$$

Noting the term from the boundary in $(4.3)^{\prime}$, we add $\lambda \int_{0}^{t} \int_{0}^{\infty}(4.6) d x d \tau$ to $(4.3)^{\prime}$ for a small constant $\lambda(0<\lambda \ll 1)$ to obtain

$$
\begin{aligned}
C^{-1}\left(\|\psi(t)\|^{2}+\int_{0}^{\infty}\left\{V(t, x)^{1-\gamma} \widetilde{\Phi}(\tilde{v}(t, x))+\left(\frac{\tilde{v}_{x}}{\tilde{v}}\right)^{2}(t, x)\right\} d x\right) \\
+C^{-1} \int_{0}^{t} \int_{0}^{\infty}\left\{\frac{V_{t}}{V^{\gamma}}\left(\frac{1}{\tilde{v}^{\gamma}}-1+\gamma(\tilde{v}-1)\right)+\left|\frac{\phi \psi_{x} V_{t}}{v V}\right|\right. \\
\left.\quad+\frac{\psi_{x}^{2}}{v}+\frac{\gamma \tilde{v}_{x}^{2}}{V^{\gamma} \tilde{v}^{\gamma+2}}\right\} d x d t \\
\leq C\left(\left\|\phi_{0}\right\|_{1}^{2}+\left\|\psi_{0}\right\|^{2}+\int_{0}^{t}\left\|G_{x}(\tau)\right\|\left(\|\psi(\tau)\|+\left\|\frac{\tilde{v}_{x}}{\tilde{v}}(\tau)\right\|\right) d \tau\right) \\
+\lambda \gamma \int_{0}^{t} \int_{0}^{\infty}\left|\frac{V_{x}}{V^{\gamma+1}}\left(\frac{1}{\tilde{v}^{\gamma}}-1\right) \frac{\tilde{v}_{x}}{\tilde{v}}\right| d x d \tau \\
\quad+\left.\int_{0}^{t}\left\{(p(V+\phi)-p(V)) \psi-(\mu-\lambda)\left(\frac{\phi_{t}}{v}+V_{t}\left(\frac{1}{v}-\frac{1}{V}\right)\right) \psi\right\}\right|_{x=0} d \tau .
\end{aligned}
$$

The key point is to estimate the last two terms in (4.7). 
Step 3. (Estimate of the second to last term in (4.7)) Put

$$
I:=\lambda \gamma \int_{0}^{t} \int_{0}^{\infty}\left|\frac{V_{x}}{V^{\gamma+1}}\left(\frac{1}{\tilde{v}^{\gamma}}-1\right) \frac{\tilde{v}_{x}}{\tilde{v}}\right| d x d \tau
$$

When $\gamma=1$, simply, for small $\nu>0$,

$$
\begin{aligned}
I & \leq \int_{0}^{t} \int_{0}^{\infty}\left\{\nu \frac{\tilde{v}_{x}^{2}}{\tilde{v}^{3}}+C_{\nu} \frac{V_{x}^{2}(1-\tilde{v})^{2}}{\tilde{v}}\right\} d x d \tau \\
& \leq \int_{0}^{t} \int_{0}^{\infty}\left\{\nu \frac{\tilde{v}_{x}^{2}}{\tilde{v}^{\gamma+2}}+C_{\nu} C \varepsilon V_{t}\left(\frac{1}{\tilde{v}^{\gamma}}-1+\gamma(\tilde{v}-1)\right)\right\} d x d \tau
\end{aligned}
$$

which are both absorbed into the left-hand side of (4.7) for suitably small constants $\nu$ and $\varepsilon$. When $\gamma>1$, let $I$ be divided into three parts:

$$
\begin{aligned}
& I=\lambda \gamma \int_{0}^{t}\left(\int_{\Omega_{1}(\tau)}+\int_{\Omega_{2}(\tau)}+\int_{\Omega_{3}(\tau)}\right) d \tau \\
& \quad:=I_{1}+I_{2}+I_{3},
\end{aligned}
$$

where

$$
\begin{aligned}
& \Omega_{1}(t)=\left\{x \in \mathbf{R}_{+} ; v(t, x) \geq 2\right\} \\
& \Omega_{2}(t)=\left\{x \in \mathbf{R}_{+} ; \frac{1}{2}<v(t, x)<2\right\} \\
& \Omega_{3}(t)=\left\{x \in \mathbf{R}_{+} ; v(t, x) \leq \frac{1}{2}\right\}
\end{aligned}
$$

For suitably small $\nu>0$,

$$
\begin{aligned}
I_{3} & \leq \nu \int_{0}^{t} \int_{\Omega_{3}(\tau)} \frac{\tilde{v}_{x}^{2}}{\tilde{v}^{\gamma+2}} d x d \tau+C_{\nu} \int_{0}^{t} \int_{\Omega_{3}(\tau)} \frac{V_{x}^{2}\left(1-\tilde{v}^{\gamma}\right)^{2}}{\tilde{v}^{\gamma}} d x d \tau \\
& \leq \nu \int_{0}^{t} \int_{0}^{\infty} \frac{\tilde{v}_{x}^{2}}{\tilde{v}^{\gamma+2}} d x d \tau+C_{\nu} \int_{0}^{t} \sup _{\mathbf{R}}\left|V_{x}\right| \cdot \int_{\Omega_{3}(\tau)} V_{t}\left(\tilde{v}^{-\gamma}-1+\gamma(\tilde{v}-1)\right) d x d \tau
\end{aligned}
$$

because $\frac{\left(1-\tilde{\tilde{v}}^{\gamma}\right)^{2}}{\tilde{v}^{\gamma}} \sim \tilde{v}^{-\gamma}-1+\gamma(\tilde{v}-1)$ as $\tilde{v} \rightarrow 0$. Here, $f(x) \sim g(x)$ as $x \rightarrow a$ means that for any $x$ in the neighborhood of $a$,

$$
C^{-1} g(x) \leq f(x) \leq C g(x)
$$

Therefore, it follows that by Lemma 2.2 ,

$$
\left|I_{3}\right| \leq \nu \int_{0}^{t} \int_{0}^{\infty} \frac{\tilde{v}_{x}^{2}}{\tilde{v}^{\gamma+2}} d x d \tau+C_{\nu} C \varepsilon \int_{0}^{t} \int_{0}^{\infty} V_{t}\left(\tilde{v}^{-\gamma}-1+\gamma(\tilde{v}-1)\right) d x d \tau
$$

It is clear that

$$
\left|I_{2}\right| \leq \nu \int_{0}^{t} \int_{0}^{\infty} \frac{\tilde{v}_{x}^{2}}{\tilde{v}^{\gamma+2}} d x d \tau+C_{\nu} C \varepsilon \int_{0}^{t} \int_{0}^{\infty} V_{t}\left(\tilde{v}^{-\gamma}-1+\gamma(\tilde{v}-1)\right) d x d \tau
$$


Finally,

$$
\begin{aligned}
\left|I_{1}\right| \leq & C \int_{0}^{t} \int_{\Omega_{1}}\left|V_{x}\right| \cdot\left|\frac{\tilde{v}_{x}}{\tilde{v}}\right| d x d \tau \\
\leq & \nu \int_{0}^{t} \int_{\Omega_{1}(\tau)} V_{t} \tilde{v} d x d \tau+C_{\nu} \int_{0}^{t} \int_{\Omega_{1}(\tau)}\left|V_{x}\right| \frac{\tilde{v}_{x}^{2}}{\tilde{v}^{3}} d x d \tau \\
\leq & C \nu \int_{0}^{t} \int_{\Omega_{1}(\tau)} V_{t}\left(\frac{1}{\tilde{v}^{\gamma}}-1+\gamma(\tilde{v}-1)\right) d x d \tau \\
& +\nu \int_{0}^{t} \int_{\Omega_{1}(\tau)} \frac{\tilde{v}_{x}^{2}}{\tilde{v}^{\gamma+2}} d x d \tau+C_{\nu} \int_{0}^{t} \int_{\Omega_{1}(\tau)}\left|V_{x}\right|^{\frac{\gamma}{\gamma-1}}\left(\frac{\tilde{v}_{x}}{\tilde{v}}\right)^{2} d x d \tau,
\end{aligned}
$$

because $\tilde{v}^{-\gamma}-1+\gamma(\tilde{v}-1) \sim \tilde{v}$ as $\tilde{v} \rightarrow \infty$ and

$$
\left|V_{x}\right| \frac{\tilde{v}_{x}^{2}}{\tilde{v}^{\gamma+2}}=\frac{\left|\tilde{v}_{x}\right|^{2 / \gamma}}{\tilde{v}^{2 / \gamma+1}} \cdot\left|V_{x}\right|\left(\frac{\tilde{v}_{x}}{\tilde{v}}\right)^{2 / \gamma^{\prime}} \quad \text { for } \frac{1}{\gamma}+\frac{1}{\gamma^{\prime}}=1 .
$$

Since $\sup _{\mathbf{R}}\left|V_{x}(t, x)\right| \leq C(1+t)^{-1}$ by Lemma 2.2 , (4.12) yields

$$
\begin{aligned}
\left|I_{1}\right| \leq & C \nu \int_{0}^{t} \int_{0}^{\infty} V_{t}\left(\frac{1}{\tilde{v}^{\gamma}}-1+\gamma(\tilde{v}-1)\right) d x d \tau \\
& +\nu \int_{0}^{t} \int_{0}^{\infty} \frac{\tilde{v}_{x}^{2}}{\tilde{v}^{\gamma+2}} d x d \tau+C \int_{0}^{t}(1+\tau)^{-\frac{\gamma}{\gamma-1}} \int_{0}^{\infty}\left(\frac{\tilde{v}_{x}}{\tilde{v}}\right)^{2} d x d \tau .
\end{aligned}
$$

Thus, for small $\nu$ and $\varepsilon>0$, all terms in (4.10), (4.11), and (4.13) except for the last term in (4.13) are absorbed into the left-hand side of (4.7). The last term in (4.13) will be estimated by the Gronwall inequality.

REMARK. These estimates are still available for the Cauchy problem, so that these improve the result in [8] restricted to $1 \leq \gamma \leq 2$ to Theorem 1.2. The details will be omitted.

Step 4. (Estimates of the terms coming from the boundary) Since

$$
\begin{aligned}
\frac{\phi_{t}(t, 0)}{v(t, 0)} & =\frac{d}{d t} \log v(t, 0)-\frac{V_{t}(t, 0)}{v(t, 0)} \\
& =\frac{d}{d t} \log \frac{v(t, 0)}{V(t, 0)}-V_{t}(t, 0)\left(\frac{1}{v(t, 0)}-\frac{1}{V(t, 0)}\right),
\end{aligned}
$$

$$
\begin{aligned}
J:= & \text { the last term in }(4.7) \\
= & \int_{0}^{t}\left\{\left(\frac{1}{v(\tau, 0)^{\gamma}}-\frac{1}{V(\tau, 0)^{\gamma}}\right) \psi(\tau, 0)-(\mu-\lambda)\left(\frac{d}{d t} \log \frac{v(\tau, 0)}{V(\tau, 0)}\right) \psi(\tau, 0)\right\} d \tau \\
= & -(\mu-\lambda) \log \frac{v(t, 0)}{V(t, 0)} \cdot \psi(t, 0) \\
& +\int_{0}^{t}\left\{(\mu-\lambda) \log \frac{v(\tau, 0)}{V(\tau, 0)} \cdot \psi_{t}(\tau, 0)+\left(\frac{1}{v(\tau, 0)^{\gamma}}-\frac{1}{V(\tau, 0)^{\gamma}}\right) \psi(\tau, 0)\right\} d \tau \\
:= & J_{1}(t)+\int_{0}^{t} J_{2}(\tau) d \tau .
\end{aligned}
$$


Similar to the preceding step, we divide into three cases. For suitably small $v_{1}\left(\leq v_{+}<\right.$ $\left.V(t, 0)<v_{-}\right)$, when $v(t, 0) \leq v_{1}$ or $v(\tau, 0) \leq v_{1}$,

$$
\begin{gathered}
-(\mu-\lambda) \log \frac{v(t, 0)}{V(t, 0)} \cdot \psi(t, 0)<0 \\
(\mu-\lambda) \log \frac{v(\tau, 0)}{V(\tau, 0)} \cdot \psi_{t}(\tau, 0)<0 \\
\left(\frac{1}{v(\tau, 0)^{\gamma}}-\frac{1}{V(\tau, 0)^{\gamma}}\right) \psi(\tau, 0)<0
\end{gathered}
$$

because $\psi(t, 0)<0$ and $\psi_{t}(t, 0)>0$. Hence all terms can be dropped. Next, in order to treat the case of large $v(t, 0)$, we introduce

$$
\Psi(\tilde{v})=\int_{0}^{\tilde{v}} \widetilde{\Phi}(\eta)^{1 / 2} \frac{d \eta}{\eta}
$$

which is equivalent to $\tilde{v}^{1 / 2}$ as $\tilde{v} \rightarrow \infty$. (Functions of this type were first introduced by $\mathrm{Kanel}^{\prime}[1]$.) Hence, when $v(t, 0) \geq v_{2}$ for suitably large $v_{2}\left(\geq v_{-}>V(t, 0)>v_{+}\right)$,

$$
\begin{aligned}
& \left|-(\mu-\lambda) \log \frac{v(t, 0)}{V(t, 0)}\right| \leq \Psi(\tilde{v}(t, 0)), \\
& \left|\frac{1}{v(t, 0)^{\gamma}}-\frac{1}{V(t, 0)^{\gamma}}\right| \leq \Psi(\tilde{v}(t, 0)),
\end{aligned}
$$

and

$$
\begin{aligned}
\Psi(\tilde{v}(t, 0)) & =\left|\int_{0}^{\infty} \frac{\partial}{\partial x} \Psi(\tilde{v}(t, x)) d x\right| \\
& =\left|\int_{0}^{\infty} \widetilde{\Phi}(\tilde{v}(t, x))^{1 / 2} \frac{\tilde{v}_{x}(t, x)}{\tilde{v}(t, x)} d x\right| \\
& \leq \frac{1}{2} \int_{0}^{\infty}\left\{\widetilde{\Phi}(\tilde{v}(t, x))+\left(\frac{\tilde{v}_{x}}{\tilde{v}}\right)^{2}(t, x)\right\} d x
\end{aligned}
$$

Inequalities (4.15)-(4.18) yield

$$
\begin{aligned}
J_{1}(t) & =-(\mu-\lambda) \log \frac{v(t, 0)}{V(t, 0)} \cdot \psi(t, 0) \\
& \leq \begin{cases}0 & \text { if } v(t, 0) \leq v_{1}, \\
C_{v_{1}, v_{2}}|\psi(t, 0)| & \text { if } v_{1}<v(t, 0)<v_{2}, \\
\frac{\mu-\lambda}{2}|\psi(t, 0)| \int_{0}^{\infty}\left\{\tilde{\Phi}(\tilde{v}(t, x))+\left(\frac{\tilde{v}_{x}}{\tilde{v}}\right)^{2}(t, x)\right\} d x & \text { if } v(t, 0) \geq v_{2},\end{cases}
\end{aligned}
$$


and

$$
\begin{aligned}
\int_{0}^{t} J_{2}(\tau) d \tau= & \int_{\left\{v(\tau, 0) \leq v_{1}\right\}}+\int_{\left\{v_{1}<v(\tau, 0)<v_{2}\right\}}+\int_{\left\{v(\tau, 0) \geq v_{2}\right\}} \\
\leq & \int_{0}^{t} C_{v_{1}, v_{2}}\left(\left|\psi_{t}(\tau, 0)\right|+|\psi(\tau, 0)|\right) d \tau \\
& +\int_{0}^{t} \frac{\left|\psi_{t}(\tau, 0)\right|+|\psi(\tau, 0)|}{2} \int_{0}^{\infty}\left\{\tilde{\Phi}(\tilde{v}(\tau, x))+\left(\frac{\tilde{v}_{x}}{\tilde{v}}\right)^{2}(\tau, x)\right\} d x d \tau .
\end{aligned}
$$

Here, remember that $\psi(t, 0)$ and $\psi_{t}(t, 0)$ are depending on $t_{0}$ and tend to 0 as $t_{0} \rightarrow \infty$, and are integrable in $t$ for large $q$, by virtue of (3.3) and (3.4).

Thus, combining (4.7), (4.13), (4.14), (4.19), and (4.20) in Steps 2-4 and using the Gronwall inequality, we obtain the following lemma.

Lemma 4.2 (Basic Estimates). For suitably small $\varepsilon>0$ and large $t_{0}, q>0$, it follows that

$$
\begin{aligned}
\|\psi(t)\|^{2} & +\int_{0}^{\infty}\left\{\widetilde{\Phi}(\tilde{v}(t, x))+\left(\frac{\tilde{v}_{x}}{\tilde{v}}\right)^{2}(t, x)\right\} d x \\
& +\int_{0}^{t} \int_{0}^{\infty}\left\{V_{t}\left(\frac{1}{\tilde{v}^{\gamma}}-1+\gamma(\tilde{v}-1)\right)+\left|\frac{\phi \psi_{x} V_{t}}{v V}\right|+\frac{\psi_{x}^{2}}{v}+\frac{\tilde{v}_{x}^{2}}{\tilde{v}^{\gamma+1}}\right\} d x d \tau \\
\leq & C\left(\left\|\phi_{0}\right\|_{1}^{2}+\left\|\psi_{0}\right\|^{2}+\left|\left(\phi_{0}, \psi_{0}\right)(0)\right|^{2}+1\right) .
\end{aligned}
$$

Step 5. Following $\mathrm{Kanel}^{\prime}$ [1], we now have the key lemma.

LEMmA 4.3. It follows that for some constant $C$ independent of $T$,

$$
C^{-1} \leq v=V+\phi \leq C .
$$

Proof. Since

$$
\Psi(\tilde{v}) \rightarrow \begin{cases}-\infty & \text { as } \tilde{v} \rightarrow 0+ \\ +\infty & \text { as } \tilde{v} \rightarrow+\infty\end{cases}
$$

and, similar to (4.17),

$$
\begin{aligned}
|\Psi(\tilde{v}(t, x))| & =\left|\int_{x}^{\infty} \frac{\partial}{\partial x} \Psi(\tilde{v}(t, x)) d x\right| \\
& \leq \frac{1}{2} \int_{0}^{\infty}\left\{\tilde{\Phi}(\tilde{v}(t, x))+\left(\frac{\tilde{v}_{x}}{\tilde{v}}\right)^{2}(t, x)\right\} d x,
\end{aligned}
$$

(4.22) follows from (4.21).

By virtue of $(4.22),(4.21)$ is rewritten by the inequalities of $\phi, \phi_{x}, \psi, \psi_{x}$ as follows:

LEMMA 4.4. It follows that

$$
\begin{gathered}
\|\phi(t)\|_{1}^{2}+\|\psi(t)\|^{2}+\int_{0}^{t}\left(\left\|\sqrt{V_{t}} \phi(\tau)\right\|^{2}+\left\|\left(\phi_{x}, \psi_{x}\right)(\tau)\right\|^{2}\right) d \tau \\
\leq C\left(\left\|\phi_{0}\right\|_{1}^{2}+\left\|\psi_{0}\right\|^{2}+\left|\left(\phi_{0}, \psi_{0}\right)(0)\right|^{2}+1\right) .
\end{gathered}
$$


Step 6. (Higher-order estimate) Making use of Lemma 4.3, we rewrite $(3.1)_{2}$ as

$$
\psi_{t}-\mu \frac{\psi_{x x}}{V+\phi}=G_{x}+H
$$

where

$$
H=O\left(\left(V_{x}+U_{x x}\right) \phi, \phi_{x}, \psi_{x}, \phi_{x} \psi_{x}\right) .
$$

Multiplying (4.24) by $-\psi_{x x}$, we have

$$
\left(\frac{1}{2} \psi_{x}^{2}\right)_{t}-\left(\psi_{t} \psi_{x}\right)_{x}+\mu \frac{\psi_{x x}^{2}}{V+\phi}=-\left(G_{x}+F\right) \psi_{x x} .
$$

The value from the boundary after integration of (4.26) in $x$ is estimated as

$$
\left|\psi_{t} \psi_{x}(t, 0)\right| \leq \nu\left\|\psi_{x x}(t)\right\|^{2}+C\left(\left\|\psi_{x}(t)\right\|^{2}+\left|\psi_{t}(t, 0)\right|^{2}\right) .
$$

In the final terms, for example, by (4.23)

$$
\begin{gathered}
\left|\int V_{x} \phi \psi_{x x} d x\right| \leq \nu\left\|\psi_{x x}(t)\right\|^{2}+C\|\phi(t)\|^{2} \sup _{\mathbf{R}_{+}}\left|V_{x}(t, x)\right|^{2} \\
\leq \nu\left\|\psi_{x x}(t)\right\|^{2}+C(1+t)^{-2} \\
\left|\int \phi_{x} \psi_{x} \psi_{x x} d x\right| \leq \sup _{\mathbf{R}_{+}}\left|\psi_{x}(t, x)\right| \cdot\left\|\phi_{x}(t)\right\|\left\|\psi_{x x}(t)\right\| \\
\leq \nu\left\|\psi_{x x}(t)\right\|^{2}+C\left\|\psi_{x}(t)\right\|^{2} .
\end{gathered}
$$

Thus, we arrive at

LEMMA 4.5. It follows that

$$
\left\|\psi_{x}(t)\right\|^{2}+\int_{0}^{t}\left\|\psi_{x x}(\tau)\right\|^{2} d \tau \leq C\left(\left\|\phi_{0}, \psi_{0}\right\|_{1}^{2}+1\right) .
$$

Here we have used $\left|\left(\phi_{0}, \psi_{0}\right)(0)\right|^{2} \leq C\left\|\phi_{0}, \psi_{0}\right\|_{1}^{2}$.

Combining Lemma 4.4 with Lemma 4.5 we complete the proof of Proposition 3.2.

\section{REFERENCES}

[1] Ya. Kanel', On a model system of one-dimensional gas motion (Russian), Differencial'nya Uravnenija 4, 374-380 (1968)

[2] O. A. Ladyzenskaja, V. A. Solonikov, and N. N. Ural'ceva, Linear and quasilinear equations of parabolic type, Translations of Math. Mono. 23, Amer. Math. Soc., Providence, RI, 1968

[3] T.-P. Liu, A. Matsumura, and K. Nishihara, Behaviors of solutions for the Burgers equation with boundary corresponding to rarefaction waves, SIAM J. Math. Anal. 29, 293-308 (1998)

[4] T.-P. Liu and K. Nishihara, Asymptotic behavior for scalar viscous conservation laws with boundary effect, J. Differential Equations 133, 296-320 (1997)

[5] T.-P. Liu and S.-H. Yu, Propagation of a stationary shock layer in the presence of a boundary, Arch. Rat. Mech. Anal. 139, 57-82 (1997)

[6] A. Matsumura and M. Mei, Convergence to travelling fronts of solutions of the p-system with viscosity in the presence of a boundary, Arch. Rat. Mech. Anal. 146, 1-22 (1999)

[7] A. Matsumura and K. Nishihara, Asymptotics toward the rarefaction waves of the solutions of a one-dimensional model system for compressible viscous gas, Japan J. Appl. Math. 3, 1-13 (1986)

[8] A. Matsumura and K. Nishihara, Global stability of the rarefaction wave of a one-dimensional model system for compressible viscous gas, Commun. Math. Phys. 144, 325-335 (1992)

[9] T. Pan, H. Liu, and K. Nishihara, Asymptotic stability of the rarefaction wave of a one-dimensional model system for compressible viscous gas with boundary, Japan J. Indust. Appl. Math., in press 